\title{
Evidencia clínica de tracoma en indígenas colombianos del departamento de Vaupés
}

\author{
Hollman Miller ${ }^{1}$, Germán Gallego², Gerzaín Rodríguez ${ }^{3}$ \\ 1 Programa de Enfermedades Transmitidas por Vectores, Secretaría Departamental de Salud, Mitú, Vaupés, \\ Colombia \\ 2 Programa de Vigilancia en Salud Pública, Secretaría Departamental de Salud del Vaupés, Mitú, Vaupés, \\ Colombia; actualmente: Promoción y Prevención, IPS Virrey Solís, Bogotá, D.C., Colombia \\ ${ }^{3}$ Grupo de Microbiología Molecular, Facultad de Medicina, Universidad de La Sabana, Chía, Cundinamarca, \\ Colombia
}

Introducción. El tracoma es la primera causa infecciosa de ceguera. En 2008 había 1'300.000 ciegos por su causa y 8 millones de personas tenían lesiones oculares que podrían conducirlos a la ceguera. En América Latina se ha documentado su presencia en Brasil, Guatemala y México.

Objetivo. Informar por primera vez la presencia de tracoma en Colombia, en indígenas del departamento del Vaupés.

Materiales y métodos. En 2003 y 2006 se visitaron las comunidades makú de San Joaquín y Santa Catalina, a 5 km de Brasil, y entre 2007 y 2009, las de San Gerardo, San Gabriel y Nuevo Pueblo, a $35 \mathrm{~km}$ de las anteriores.

Resultados. En 2006, se examinaron 114 personas en San Joaquín y Santa Catalina. Se diagnosticó clínicamente tracoma en 21 (18,4\%), 15 de ellas (13,2\%) menores de 15 años. Se detectaron todas las fases de la enfermedad. Tres mujeres tenían opacidad corneal con notoria disminución de la agudeza visual. En las tres últimas comunidades se detectaron tres mujeres con tracoma avanzado, con opacidad corneal y ceguera. La vivienda precaria, la carencia de agua potable y de sistemas adecuados de disposición de los excrementos, y la abundancia de moscas domésticas del género Hippelates, constituyen factores de riesgo para la transmisión del tracoma.

Discusión. El tracoma existe en Colombia, con incidencia frecuente en las comunidades estudiadas. Su localización definida hace posible su eliminación en programas de salud pública. Es necesario realizar una búsqueda del tracoma en otras comunidades indígenas del Vaupés con condiciones de vida similares.

Palabras clave: Chlamydia, tracoma, moscas, población indígena, indios suramericanos, conjuntiva, factores de riesgo.

\section{Clinical evidence of trachoma in Colombian Amerindians of the Vaupés Province}

Introduction: Trachoma is the leading cause of infectious blindness in the world. In 2008 there were $1,300,000$ persons with blindness caused by trachoma and 8 million with trichiasis, which might eventually lead to blindness. In Latin America it has been documented in Brazil, Guatemala and México.

Objective: To inform the presence of trachoma for the first time in Colombia, amongst Amerindians of the Department of Vaupés.

Materials and methods: In 2003 and 2006 the Amerindian Makú communities of San Joaquín and Santa Catalina, located $5 \mathrm{~km}$ from the border with Brazil, were visited. From 2007 to 2009, San Gerardo, San Gabriel and Nuevo Pueblo, at a $35 \mathrm{~km}$ distance from San Joaquín were visited.

Results: In 2006114 people were examined in Santa Catalina and San Joaquin; 21 patients were clinically diagnosed with trachoma (18.4\%), 15 (13.2\%) of them children under 15 years old. All trachoma phases were observed. Three women had corneal opacity with poor vision. In the remaining three communities, three women with advanced trachoma with corneal opacity and blindness were detected. The poor quality of living conditions without fresh water and adequate sanitary disposal systems, and the abundance of flies identified as Hippelates sp., are risk factors for the transmission of the disease. Discussion: Trachoma exists in Colombia, and it is frequent among the studied communities. Its focalized distribution makes it amenable to elimination. It is advisable to search for trachoma in other indigenous communities in Vaupés with similar living conditions.

Key words: Chlamydia, trachoma, indigenous population; indians, South American; conjunctiva, Vaupés, flies, risk factors. 
El tracoma es una enfermedad bacteriana crónica producida por Chlamydia trachomatis, que afecta la conjuntiva y la córnea. Es la primera causa de ceguera infecciosa prevenible en el mundo (1-4). Existe en 57 países (3), entre los cuales no está incluida Colombia. En América Latina existe en México, Guatemala y Brasil (3). Es una de las 14 enfermedades tropicales olvidadas, o desatendidas, que afectan a personas que viven en condiciones de pobreza extrema, abandonadas por los programas generales de salud y de desarrollo social (5).

El objetivo de este trabajo es presentar las observaciones preliminares que nos indican que el tracoma es un problema importante en, al menos, cinco comunidades indígenas del departamento colombiano de Vaupés, entre las cuales la pobreza, la carencia de agua potable y de sistemas adecuados de disposición de los excrementos, y la abundancia de las moscas del género Hippelates, incriminadas como transmisoras de Chlamydia sp. (6), son factores que propician su presencia.

\section{Materiales y métodos}

\section{Área del estudio}

El departamento de Vaupés está situado al suroriente colombiano, en límites con Brasil (figura 1). Tiene 35.000 habitantes, $95 \%$ de los cuales son indígenas. El clima es cálido y húmedo y la mayor parte del departamento es selvático con ríos caudalosos. No hay agua potable ni luz eléctrica en los asentamientos o comunidades en los que viven varios grupos étnicos, en número que rara vez excede los 170 habitantes (figura 2), algunos seminómadas, que no hablan español y tienden a rechazar las intervenciones del "hombre blanco" en sus comunidades. No hay carreteras y el transporte se hace por vía fluvial o en avionetas monomotor. Las dos comunidades más ampliamente estudiadas viven entre $5 \mathrm{~km}$ y $6 \mathrm{~km}$ de la frontera con Brasil (figura 1).

\section{Comunidades con sospecha de tracoma}

En el año 2001, funcionarios del programa de Enfermedades Transmitidas por Vectores hicieron el hallazgo de altas densidades de moscas Hippelates en algunas comunidades indígenas

\section{Correspondencia:}

Gerzaín Rodríguez, Grupo de Microbiología Molecular, Facultad de Medicina, Universidad de La Sabana, Chía, Cundinamarca, Colombia

gerzaín_rodriguez@yahoo.com

Recibido: 19/07/10; aceptado:30/08/10 limítrofes con Brasil, de dos personas ciegas y de otras con lesiones palpebrales, que concuerdan con la forma grave del tracoma. A partir de este momento se despertó el interés por realizar acciones de vigilancia de dicho evento en la población en riesgo, pero las autoridades locales de salud no realizaron actividades concretas.

En marzo de 2003 se realizó el primer desplazamiento multidisciplinario a las comunidades de Santa Catalina (N $0^{\circ} 21^{\prime} 47,42^{\prime \prime}$, W $70^{\circ} 5^{\prime}$ 59,50") y San Joaquín (N $0^{\circ} 28^{\prime} 11,95^{\prime \prime}$, W $70^{\circ} 5^{\prime}$ 29,35'), vecinas de comunidades brasileras entre las cuales el tracoma es un problema importante de salud pública (7).

Por medio del examen clínico hecho por un biólogo y un médico, sin adiestramiento especial en tracoma diferente de las lecturas de manuales y de estudio de información accesible a través de internet, se sospechó la infección en 13 personas. Sin embargo, hubo dudas tanto del médico examinador como del servicio local de salud, sobre si realmente se trataba de tracoma. No se adelantó ningún otro estudio.

En 2006 los promotores indígenas de salud detectaron nuevamente alteraciones oculares sospechosas de tracoma en indígenas de Santa Catalina y San Joaquín. Se organizaron visitas médicas para confirmar la naturaleza de estas lesiones. El equipo de salud, encabezado por dos de nosotros (HM y GG), con conocimientos teóricos sobre el tracoma, visitó las comunidades de San Joaquín y Santa Catalina con el fin de confirmar la presencia de tracoma. Se hicieron dos reuniones comunitarias para ilustrarlos sobre la enfermedad y la manera de prevenirla y se hicieron visitas domiciliarias para actualizar el censo de población y examinar clínicamente a los pobladores.

Se determinó la agudeza visual con la técnica de Snellen y se practicó el examen externo del ojo para detectar alteraciones tales como secreción e inyección conjuntival, prolongación de los ángulos oculares, proliferación del paño vascular y "triquiasis". También se hizo oftalmoscopia directa para detectar opacidad corneal. Se hizo eversión palpebral con hisopos y se examinó la conjuntiva del tarso con lupa de 2,5X.

Se buscaron los signos cardinales del tracoma, pues los cambios que la enfermedad produce en el ojo tienen fases reconocibles clínicamente, que constituyen una forma aceptada de diagnóstico, con alta sensibilidad y que son (8-12): 


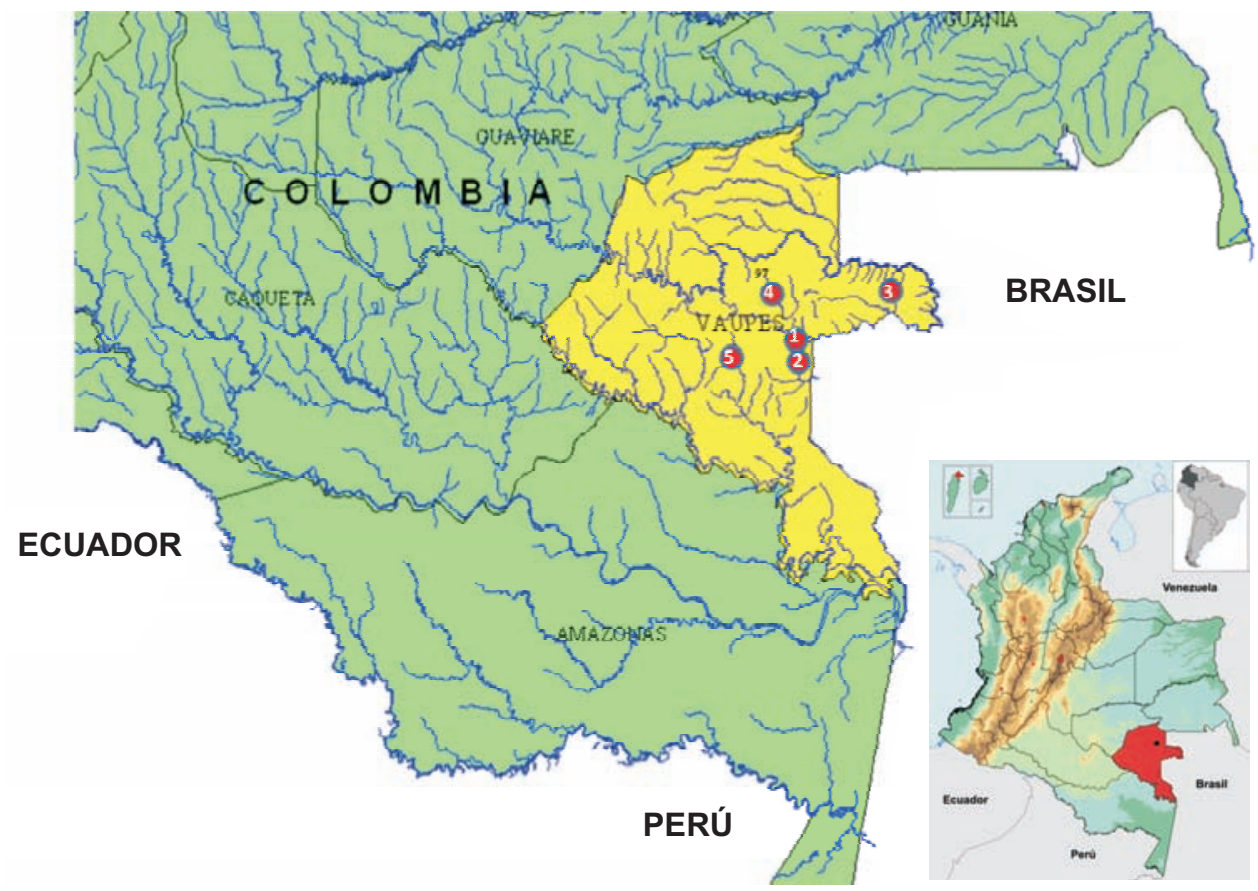

Figura 1. Área geográfica del estudio. En el departamento de Vaupés se marcan con rojo y se numeran las comunidades afectadas por tracoma: 1, San Joaquín; 2, Santa Catalina; 3, Pueblo Nuevo; 4, San Gerardo, y 5, San Gabriel.

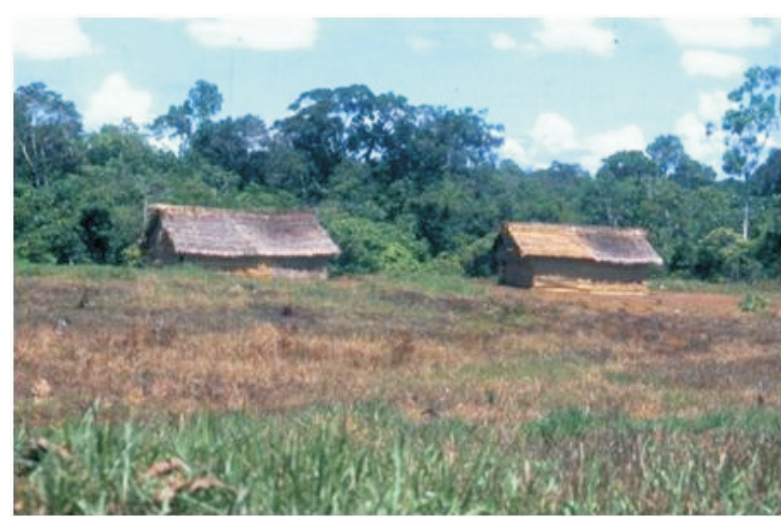

Figura 2. Viviendas de la comunidad de Santa Catalina.

- Inflamación folicular por tracoma: presencia de cinco o más folículos en la conjuntiva del tarso de no menos de $0,5 \mathrm{~mm}$.

- Inflamación intensa por tracoma: edema pronunciado de la conjuntiva del tarso superior que vela más de la mitad de sus vasos profundos.

- Cicatrices por tracoma: presencia de líneas o bandas fibrosas en la conjuntiva del tarso superior.

- Triquiasis por tracoma: como mínimo, una pestaña incrustada en el globo ocular.
- Opacidad corneal: opacidad visible de la córnea.

No se tomaron frotes ni muestras de la conjuntiva para estudio bacteriológico por carencia de los materiales adecuados.

Se examinaron las personas que en el momento del estudio estaban presentes en las comunidades, que fueron principalmente los niños y las mujeres. Los hombres jóvenes y los adultos se desplazan al área selvática o a los ríos en búsqueda de alimentos, producto de la pesca y la caza. Además, los habitantes tienden a rechazar la intervención del "hombre blanco" y no hablan español. La compañía y ayuda de un promotor comunitario fueron esenciales para realizar estas actividades.

Las lesiones oculares se fotografiaron y algunas de ellas se ilustran en las figuras 3-9.

\section{Aspectos éticos}

El estudio se realizó con el fin de verificar la presencia de las alteraciones oculares en las comunidades mencionadas y de constatar, por examen clínico, si eran debidas a tracoma. Los objetivos y procedimientos se explicaron a las comunidades a través del traductor antes de la intervención y se obtuvo su consentimiento verbal. Se hizo sólo un examen clínico, sin toma 


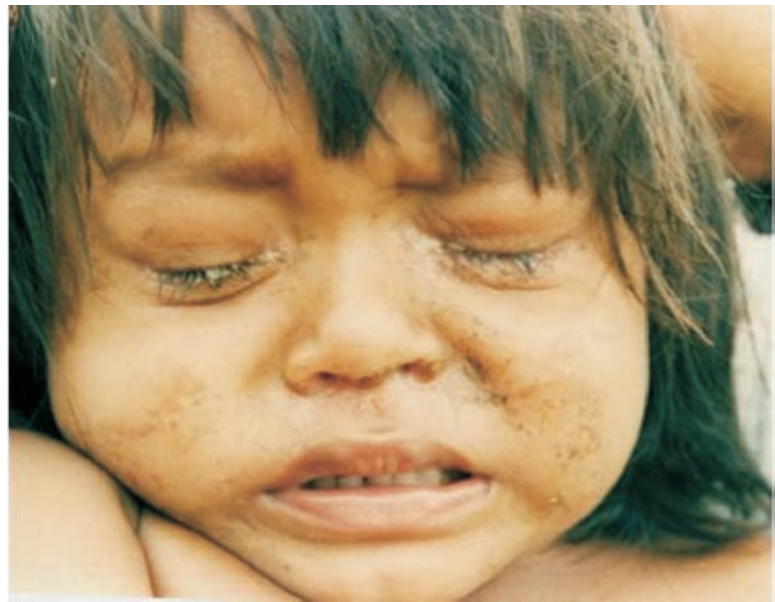

Figura 3. Secreción conjuntival mucoserosa y desaseo global de la cara.

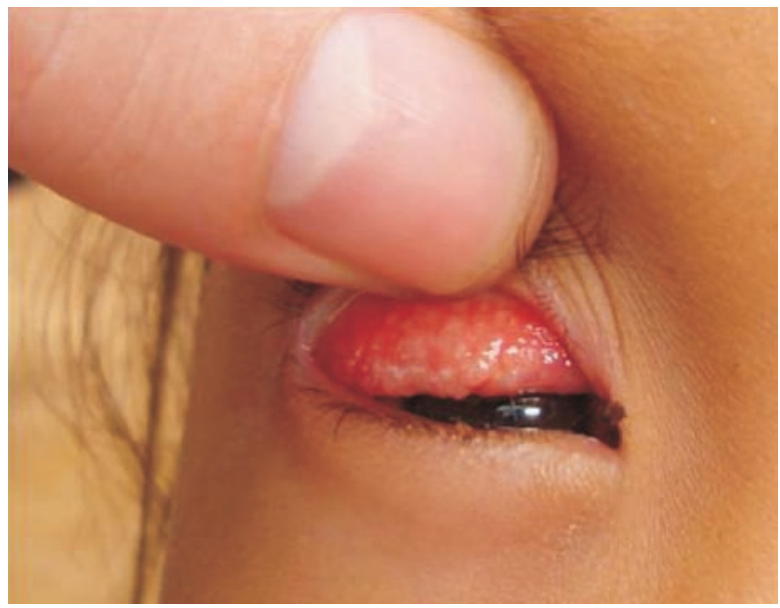

Figura 5. Folículos blanquecinos de inflamación intensa por tracoma, obliteración de la circulación venosa en la conjuntiva del tarso y presencia de Hippelates sp. en el ángulo interno del ojo.

de muestras ni procedimientos invasivos. En las comunidades de San Joaquín y Santa Catalina, la población presente en el momento de la visita se trató con azitromicina (13-16).

\section{Resultados}

Las comunidades de Santa Catalina y San Joaquín tienen en conjunto 180 habitantes, 72 adultos y 108 menores de 15 años, de la etnia jupdá-makú, que se ubican entre dos pequeños ríos o caños, afluentes de los ríos Papuri y Tiquié respectivamente, con costumbres seminómadas. San Joaquín cuenta con una alfabetizadora y un promotor de salud que han logrado un gran impacto en la prevención de enfermedades parasitarias, principalmente.

En conjunto, se examinaron las 114 personas presentes en las dos comunidades. No se

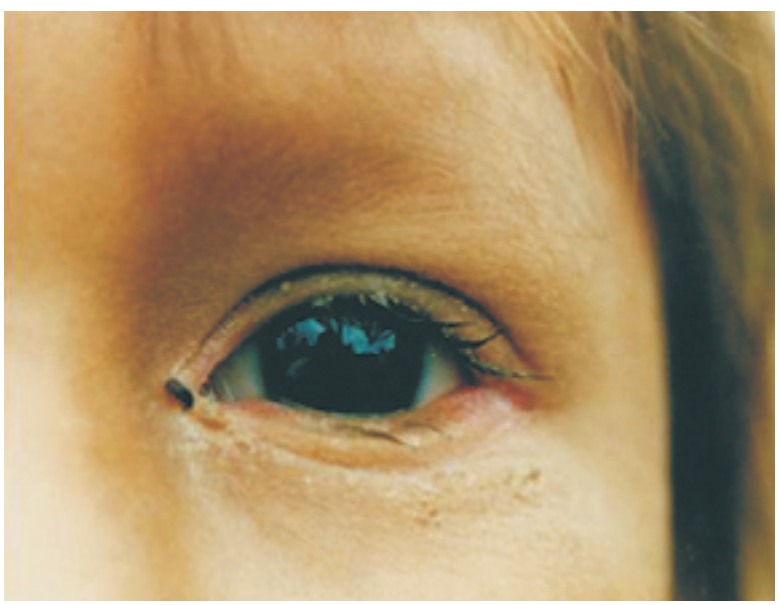

Figura 4. Hippelates sp. adherida a la secreción palpebral

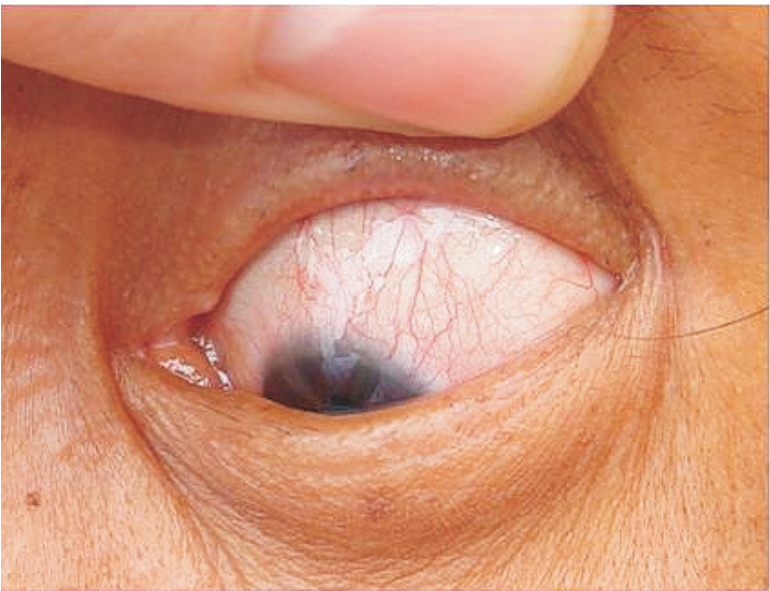

Figura 6. Proliferación de la queratitis vascular, o paño vascular, y madarosis (pérdida de las pestañas)

examinaron los menores de un año. El $40 \%$ de la población examinada provenía de la comunidad de San Joaquín (46/114). El 57\% eran mujeres (65/114) y 46\% eran menores de 15 años (53/114). Sólo 12 personas eran mayores de 50 años, 7 hombres y 5 mujeres (cuadro 1).

Se diagnosticaron 21 personas con tracoma (figuras 3 a 9), correspondiente al 18,4\% de los examinados. En el grupo de 5 a 14 años se encontraron 13 afectados, que correspondían al $61 \%$ de los casos detectados y a una prevalencia de $33,3 \%$ en este grupo de edad. En 16 individuos $(14,0 \%)$ se evidenciaron formas agudas, con inflamación intensa, incluso folicular, por tracoma (figuras 3 a 9), 15 de ellas en el grupo de menores de 1 a 14 años (prevalencia de 13,2\% en este grupo de edad) (cuadro 1). 


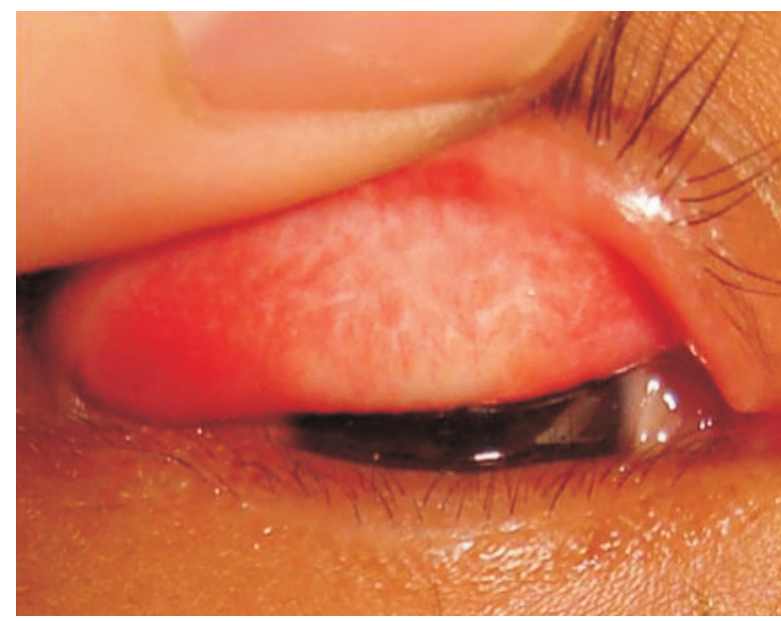

Figura 7. Cicatrices por tracoma, irregulares y blanquecinas.

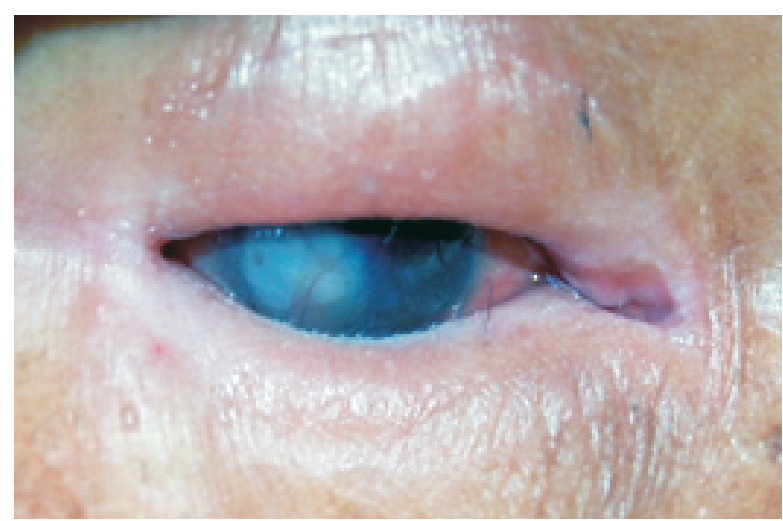

Figura 9. Triquiasis y opacidad corneal notoria en una mujer.

En los niños encontramos síntomas oculares importantes consistentes en secreción conjuntival, conjuntivitis, folículos palpebrales y queratitis vascular, o paño vascular (figuras 3 a 6), mientras que en los adultos observamos cicatrices, triquiasis y opacidad de la córnea (figuras 2 a 6 , cuadro 1). Detectamos todas las fases del tracoma, entre ellas tres mujeres con alteración importante de la

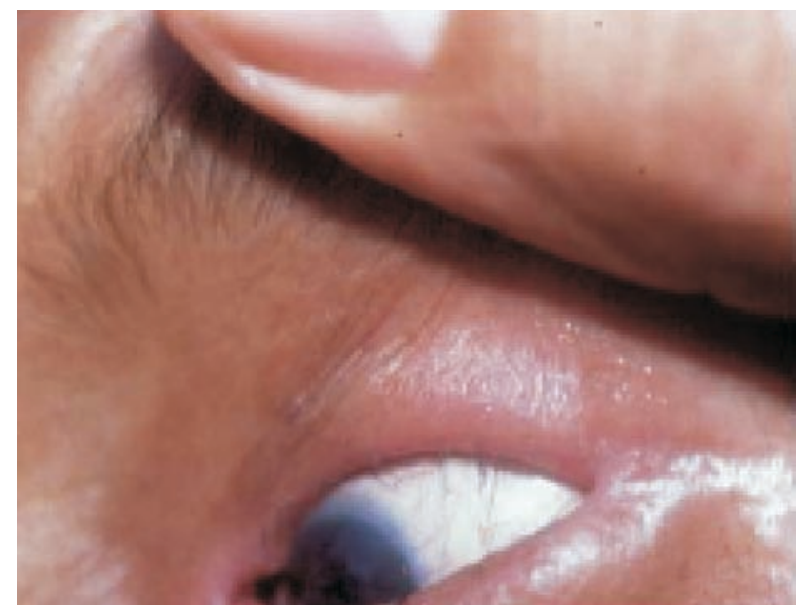

Figura 8. Triquiasis (orientación de las pestañas superiores hacia el interior del ojo) y madarosis.

agudeza visual, por opacidad corneal y triquiasis (figuras 7 a 9 , cuadro 1).

Un hecho importante fue la frecuente presencia de moscas diminutas que se posaban en las secreciones conjuntivales de los niños y que clasificamos como Chloropidos pertenecientes a los géneros Hippelates y Liohippelates (figuras 4 y 5).

Se distribuyó tratamiento con azitromicina a las 120 personas presentes de las dos comunidades, a la dosis de $20 \mathrm{mg} / \mathrm{kg}$ de peso (máximo $1 \mathrm{~g}$ ), en los individuos mayores de seis meses. En tres niños menores de seis meses, se instruyó a las madres y a las promotoras de salud para administrar tratamiento tópico ocular con ungüento de oxitetraciclina y polimixina, durante seis semanas (13-16). También se promovió el lavado de la cara y de las manos, con conferencias a los niños y a las madres de las comunidades y se enfatizó la importancia de esta práctica, que se recomendó promover a los encargados de los programas de salud comunitaria.

Cuadro 1. Distribución por grupos de edad de la población examinada y de los tipos encontrados de lesiones por tracoma.

\begin{tabular}{|c|c|c|c|c|c|c|c|}
\hline & \multicolumn{6}{|c|}{ Edad (años) } & \multirow[b]{2}{*}{$\begin{array}{c}\text { Total } \\
\mathrm{N}\end{array}$} \\
\hline & $\begin{array}{r}1 \text { a } 4 \\
n\end{array}$ & $\begin{array}{c}5 \text { a } 14 \\
n\end{array}$ & $\begin{array}{c}15 \text { a } 24 \\
n\end{array}$ & $\begin{array}{c}25 \text { a } 44 \\
n\end{array}$ & $\begin{array}{c}45 \text { a } 49 \\
n\end{array}$ & $\begin{array}{c}50 \text { y más } \\
n\end{array}$ & \\
\hline Población examinada & 14 & 39 & 19 & 24 & 6 & 12 & 114 \\
\hline Inflamación folicular por tracoma & 2 & 9 & 1 & 0 & 0 & 0 & 12 \\
\hline Inflamación intensa por tracoma & 0 & 4 & 0 & 0 & 0 & 0 & 4 \\
\hline Cicatrices por tracoma & 0 & 0 & 0 & 2 & 0 & 0 & 2 \\
\hline Triquiasis por tracoma & 0 & 0 & 0 & 0 & 1 & 0 & 1 \\
\hline Opacidad corneal & 0 & 0 & 0 & 0 & 0 & 2 & 2 \\
\hline Total de casos & 2 & 13 & 1 & 2 & 1 & 2 & 21 \\
\hline
\end{tabular}


En los años 2007, 2008 y 2009, durante las visitas anuales de vigilancia de otras enfermedades transmitidas por vectores, se registraron casualmente otros tres casos de tracoma en mujeres adultas, con la forma más grave de la enfermedad, consistente en triquiasis con opacidad corneal y ceguera, procedentes de las comunidades de San Gabriel de Caño Colorado ( $0^{\circ} 21^{\prime} 1,57^{\prime \prime}, \mathrm{W} 70^{\circ}$ $26^{\prime} 38,06^{\prime \prime}$ ), Nuevo Pueblo (N $0^{\circ} 51^{\prime}$ 53,83”,W $69^{\circ}$ $33^{\prime} 49,35$ ”) y San Gerardo del Paca (N $0^{\circ} 46^{\prime} 0,26$ ", W $\left.70^{\circ} 17^{\prime} 20,73^{\prime \prime}\right)$, casos que reflejan una mayor distribución regional de la enfermedad. No se ha hecho un estudio global de estas comunidades para medir la prevalencia de la enfermedad.

\section{Discusión}

Chlamydia trachomatis es una bacteria diminuta, intracelular obligatoria, que se transmite de persona a persona. El agente infeccioso es un "cuerpo elemental", que se adhiere a las células epiteliales de la conjuntiva y penetra al citoplasma celular en un fagosoma, al cual no se vierten lisosomas para destruirlo $(1-4,17,18)$. Su crecimiento forma un "cuerpo reticular", que se divide cada 4 a 7 días y que es un conglomerado de bacterias que utilizan los productos celulares para sobrevivir y multiplicarse, hasta que se desintegra y genera nuevos "cuerpos elementales" que infectarán otras células y huéspedes $(18,19)$. La infección repetida o persistente produce una inflamación conjuntival crónica, característica esencial del tracoma $(1,18)$. Hay numerosas serovariedades de la bacteria y es probable que las infecciones por variedades diferentes aumenten la gravedad de la enfermedad (18-21). El tracoma es producido por la infección repetida con las serovariedades $\mathrm{A}, \mathrm{B}, \mathrm{Ba}$ y $\mathrm{C}$ $(20,21)$.

La enfermedad comienza en los niños, principalmente en aquéllos entre 2 y 5 años de edad, en los cuales tiene mayor duración (22-24), como lo vemos en los casos demostrados, $61 \%$ de los cuales eran menores de 15 años. Ellos son la fuente principal de la bacteria; la transmiten a los otros niños y a sus madres (25), principalmente, a través del contacto estrecho, como compartir camas y juegos, que permiten entrar en contacto con las secreciones oculares, o por el intercambio de utensilios contaminados o fómites, como las toallas $(1-4,17,22-24)$.

Las condiciones de vivienda inadecuada, sin agua potable ni accesible, la falta de costumbre de bañarse la cara y las manos, y la libre descarga de los excrementos, no sólo propician la transmisión de la bacteria sino la proliferación de moscas domésticas, entre las cuales se identificó la abundante presencia de moscas del género Hippelates sp. Las moscas domésticas, atraídas por las secreciones conjuntivales, pueden actuar como transmisores mecánicos de la bacteria $(6,25-28)$.

Se detectaron casos de tracoma en todas sus fases, con base en la observación clínica, seis con opacidad corneana en mujeres adultas, con notoria alteración de la agudeza visual, que llegaba a la ceguera en tres de ellas. No se realizó examen microbiológico para demostrar la presencia de $C$. trachomatis, pero esta importante actividad forma parte de un proyecto en curso.

La intervención inicial realizada buscó seguir la estrategia recomendada por la Organización Mundial de la Salud (OMS) para controlar la enfermedad en el mundo, que se identifica como SAFE, por sus siglas en inglés, citadas de manera inversa a su importancia, y que son: $S$, cirugía (surgery) necesaria para corregir la triquiasis; A, antibióticos (antibiotics), suministrados en masa, por vía oral o local, gratuita y repetida, según la prevalencia de la enfermedad; F, lavado repetido de la cara (facial washing), que elimina la bacteria Chlamydia sp. y es el mecanismo más efectivo para controlar la enfermedad, pero el más difícil de implementar por la carencia de agua y por las costumbres, actitudes y cultura de las comunidades afectadas; E, mejoramiento ambiental (enviromental improvement), que abarca el suministro de agua y de vivienda adecuadas (1-4,29). Diferentes evaluaciones de este programa han demostrado que produce un impacto significativo en el control del tracoma y de la ceguera (8-12). La meta de la OMS es eliminar la enfermedad para el año 2020 $(11,29)$.

Chlamydia trachomatis es sensible a varios antibióticos, entre ellos a la azitromicina, que se puede administrar en una dosis única, por vía oral, repetida unas dos veces al año (13-16). Las modalidades de tratamiento varían según la prevalencia de la enfermedad. Cuando es muy alta, entre $10 \%$ y $50 \%$ de la comunidad afectada, se debe suministrar a todos los miembros de la comunidad, conducta que buscamos seguir, pero que fue difícil de cumplir por el corto tiempo de nuestra estadía en las comunidades afectadas y por la tendencia migratoria de la población. Cuando la prevalencia es menor puede tratarse sólo el grupo familiar. En ningún caso es recomendable sólo el tratamiento de casos individuales (16). 
El tratamiento con azitromicina se facilita porque el antibiótico se suministra por vía oral, una o dos veces al año, no produce efectos secundarios, no ha generado resistencia y se puede obtener de forma gratuita, suministrado por los Laboratorios Pfizer, a través del Programa de Eliminación del Tracoma de la OMS $(1-4,14)$.

Este tratamiento se suministró a 120 personas. No se dio a toda la población porque no estaba presente y porque esperar su retorno es incierto y no se ajustaba al tiempo disponible de la comisión. De todas maneras, se dejó medicamento suficiente con los promotores de salud, con instrucciones de suministrarlo a las personas de la comunidad, a su regreso. A pesar de su seguridad, no se suministró a tres niños menores de seis meses de edad (16). Los pacientes con triquiasis sólo recibieron el antibiótico, pues el tratamiento quirúrgico estaba fuera de toda posibilidad.

En conclusión, por métodos clínicos, que tienen sensibilidad razonable, se detectó la presencia de todas las fases del tracoma, incluida la opacidad corneal con compromiso importante de la agudeza visual y ceguera, en cinco comunidades indígenas del departamento de Vaupés, en las cuales se diagnosticaron 21 casos, entre 114 pacientes examinados (18\%).

Es necesario confirmar los hallazgos clínicos con estudios bacteriológicos que permitan demostrar que $C$. trachomatis es el agente etiológico de las lesiones observadas. Las condiciones de vivienda precaria, sin agua potable, y la abundancia de moscas domésticas del género Hippelates, probable vector mecánico de la bacteria, son importantes factores de riesgo para su transmisión.

La localización precisa de la endemia, el poco número de habitantes y la disponibilidad de tratamiento adecuado, deben permitir la eliminación del tracoma, a través de la intervención SAFE recomendada por la OMS, que las autoridades de salud deberían implementar en la población afectada. Es necesario persistir en la búsqueda de la enfermedad en otras comunidades de la región, que tienen condiciones similares de vida.

\section{Agradecimientos}

A la Fundación Médicos sin Fronteras, a través de Stephane Doyon, que suministró gratuitamente los medicamentos usados en la atención de los pacientes. Al médico Pablo Martínez y al bacteriólogo Julián Trujillo, quienes participaron en los desplazamientos, a los promotores de salud César Tamayo y Estanislao Valle y a los auxiliares del Programa de Control de Enfermedades Trasmitidas por Vectores de la Secretaría Departamental de Salud del Vaupés, por su labor dedicada en las comunidades estudiadas.

\section{Conflicto de intereses}

Los autores declaramos que no tenemos conflictos de interés en la realización de este trabajo.

\section{Financiación}

Los gastos destinados para la realización de este estudio fueron financiados por la Secretaría de Salud del Vaupés. La Universidad de La Sabana prestó ayuda eficiente en la búsqueda bibliográfica.

\section{Referencias}

1. Mabey DC, Solomon AW, Foster A. Trachoma. Lancet. 2003;362:223-9.

2. Solomon AW, Peeling R, Foster A, Mabey DC. Diagnosis and assessment of trachoma. Clin Microbiol Rev. 2004;17:982-1011.

3. Burton MJ, Mabey DC. The global burden of trachoma: A review. PLoS Negl Trop Dis. 2009;3:e460.

4. Mathew AA, Turner A, Taylor HR. Strategies to control trachoma. Drugs. 2009;69:953-70.

5. Reddy M, Gill SS, Kalkar SR, Wu W, Anderson PJ Rochon PA. Oral drug therapy for multiple neglected tropical diseases. JAMA. 2007;298:1911-24.

6. Reilly LA, Favacho J, Garcez LM, Courtenay 0. Preliminary evidence that synanthropic flies contribute to the transmission of trachoma-causing Chlamydia trachomatis in Latin America. Cad Saúde Pública. 2007;23:1-7.

7. Monteiro de Barros O. Manual de Controle do Tracoma. Brasilia: Ministerio da Saude; 2001.

8. Emerson PM, Cairncross S, Bailey RL Mabey DC. Review of the evidence base for the " $F$ " and "E" components of the SAFE strategy for trachoma control. Trop Med Int Health. 2000;5:515-27.

9. Thein J, Zhao P, Liu H, Xu J, Jha HC, Miao Y, et al. Does clinical diagnosis indicate ocular chlamydial infection in areas with a low prevalence of trachoma? Ophthalmic Epidemiol. 2002;9:263-9.

10. Kuper H, Solomon AW, Buchan J, Zondervan M, Foster A, Mabey D. A critical review of the SAFE strategy for the prevention of blinding trachoma. Lancet Infect Dis. 2003;3:372-81.

11. Kumeresan JA, Mekaskey JW. The global elimination of blinding trachoma: progress and promise. Am J Trop Med Hyg. 2003;69(Suppl.5):24-8.

12. Miller K, Schmidt G, Melese M, Alemayehu W, Yi E, Cevallos V, et al. How reliable is the clinical exam in detecting ocular chlamydial infection? Ophthalmic Epidemiol. 2004;11:255-62. 
13. Fraser-Hurt N, Bailey RL, Cousens S, Mabey D, Faal H, Mabey DC. Efficacy of oral azithromycin versus topical tetracycline in mass treatment of endemic trachoma. Bull World Health Organ. 2001;79:632-40.

14. Senn L, Hammerschlag MR, Greub G. Therapeutic approaches to Chlamydia infections. Expert Opin Pharmacother. 2005;6:2281-90.

15. West SK, Munoz B, Mkocha H, Aguirre A, Solomon AW, Foster A, et al. Infection with Chlamydia trachomatis after mass treatment of a trachoma hyperendemic community in Tanzania: a longitudinal study. Lancet. 2005;366:1296-300.

16. Schémann JF, Guinot C, Traore L, Zefack G, Dembele M, Diallo I, et al. Longitudinal evaluation of three azithromycin distribution strategies for treatment of trachoma in a SubSaharan African country, Mali. Acta Trop. 2007;101:40-53.

17. Arango Al, Máttar S, Visbal VJ. Chlamydia trachomatis: aspectos microbiológicos, clínicos y epidemiológicos. MVZCórdoba. 2001;6:87-96.

18. Kalayoglu MV. Ocular chlamydial infections: Pathogenesis and emerging treatment strategies. Curr Drug Targets Infect Disord. 2002;2:85-91.

19. Faal N, Bailey RL, Sarr I, Joof H, Mabey DC, Holland MJ. Temporal cytokine gene expression patterns in subjects with trachoma identify distinct conjuntival responses associated with infection. Clin Exp Immunol. 2005;142:347-53.

20. Zhang J, Lietman T, Olinger L, Miao Y, Stephens R. Genetic diversity of Chlamydia trachomatis and the prevalence of trachoma. Pediatr Infect Dis J. 2004;23:21720.

21. Porter M, Mak D, Chidlow G, Harnett GB, Smith DW. The molecular epidemiology of ocular Chlamydia trachomatis infections in Western Australia: implications for trachoma control. Am J Trop Med Hyg. 2008;78:514-7.

22. Grassly NC, Ward ME, Ferris S, Mabey DC, Bailey RL. The natural history of trachoma infection and disease in a Gambian cohort with frequent follow-up. PLoS Negl Trop Dis. 2008;2:e341.

23. Abdou A, Nassirou B, Kadri B, Moussa F, Munoz BE, Opong $\mathrm{E}$, et al. Prevalence and risk factors for trachoma and ocular Chlamydia trachomatis infection in Niger. $\mathrm{Br} \mathrm{J}$ Ophtalmol. 2007;91:13-7.

24. Schémann JF, Sacko D, Malvy D, Momo G, Traore L, Bore O, et al. Risk factors for trachoma in Mali. Int J Epidemiol. 2002;31:194-201.

25. Cromwell EA, Courtright P, King JD, Rotondo LA, Ngondi $\mathbf{J}$, Emerson PM. The excess burden of trachomatous trichiasis in women: a systematic review and meta-analysis. Trans R Soc Trop Med Hyg. 2009;103:985-92.

26. Forsey T, Darougar S. Transmission of chlamydiae by the housefly. Br J Ophthalmol. 1981;65:147-50.

27. Emerson PM, Bailey RL, Olaimatu SM, Walraven GE, Lindsay SW. Transmission ecology of the fly Musca sorbens, a putative vector of trachoma. Trans R Soc Trop Med Hyg. 2000;94:28-32.

28. Emerson PM, Lindsay SW, Alexander N, Bah M, Dibba S-M, Faal HB, et al. Role of flies and provision of latrines in trachoma control: cluster-randomized controlled trial. Lancet. 2004;363:1093-8.

29. World Health Organization (WHO). The SAFE strategy: Preventing trachoma. A guide for environmental sanitation and improved hygiene. WHO/PBD/GET/00.7. Geneva: WHO; 2000. 